\title{
Biosynthetic Pathway and the Potential Role of Melatonin at Different Abiotic Stressors and Developmental Stages in Tolypocladium guangdongense
}

OPEN ACCESS

Edited by:

Hua Xiang,

Institute of Microbiology,

Chinese Academy of Sciences (CAS),

China

Reviewed by:

Caihong Dong,

Chinese Academy of Sciences (CAS),

China

Chengshu Wang,

Center for Excellence in Molecular Plant Sciences, Chinese Academy of

Sciences (CAS), China

*Correspondence:

Xueshuang Huang

xueshuanghuang@126.com

Taihui Li

mycolab@263.net

Wangqiu Deng

dengwq@gdim.cn

tThese authors have contributed equally to this work

Specialty section:

This article was submitted to Microbial Physiology and Metabolism,

a section of the journal

Frontiers in Microbiology

Received: 23 July 2021 Accepted: 06 September 2021

Published: 08 October 2021

Citation:

Wang G, Chen X, Zhang C, Li M,

Sun C, Zhan N, Huang X, Li T and Deng W (2021) Biosynthetic Pathway and the Potential Role of Melatonin at

Different Abiotic Stressors and

Developmental Stages in

Tolypocladium guangdongense.

Front. Microbiol. 12:746141.

doi: 10.3389/fmicb.2021.746141
Gangzheng Wang ${ }^{1 \dagger}$, Xianglian Chen ${ }^{1,2+}$, Chenghua Zhang ${ }^{1}$, Min Li ${ }^{1,3}$, Chengyuan Sun ${ }^{1,4}$, Ning Zhan ${ }^{1,5}$, Xueshuang Huang ${ }^{2 *}$, Taihui Li $^{1 *}$ and Wangqiu Deng ${ }^{1 *}$

'Guangdong Provincial Key Laboratory of Microbial Culture Collection and Application, State Key Laboratory of Applied Microbiology Southern China, Institute of Microbiology, Guangdong Academy of Sciences, Guangzhou, China, ${ }^{2}$ Hunan Provincial Key Laboratory for Synthetic Biology of Traditional Chinese Medicine, Hunan University of Medicine, Huaihua, China, ${ }^{3}$ College of Agriculture and Animal Husbandry, Tibet University, Nyingchi, China, ${ }^{4}$ College of Plant Protection, China Agricultural University, Guangzhou, China, ${ }^{5}$ College of Bioscience and Biotechnology, Hunan Agricultural University, Changsha, China

Melatonin, a bioactive compound and an important signaling molecule produced in plants and animals, is involved in many biological processes. However, its function and synthetic pathways in fungi are poorly understood. Here, the samples from Tolypocladium guangdongense, a highly valued edible fungus with functional food properties, were collected under different experimental conditions to quantify the levels of melatonin and its intermediates. The results showed that the intracellular melatonin content was markedly improved by Congo red (CR), cold, and heat stresses; the levels of intracellular melatonin and its intermediates increased at the primordial $(\mathrm{P})$ and fruiting body (FB) stages. However, the levels of most intermediates exhibited a notable decrease under CR stress. Several genes related to melatonin synthesis, excluding AADC (aromatic-L-amino-acid decarboxylase), were markedly upregulated at an early stage of CR stress but downregulated later. Compared to the mycelial stage, those genes were significantly upregulated the $\mathrm{P}$ and $\mathrm{FB}$ stages. Additionally, exogenous melatonin promoted resistance to several abiotic stressors and $\mathrm{P}$ formation in $T$. guangdongense. This study is the first to report melatonin biosynthesis pathway in macro-fungi. Our results should help in studying the diversity of melatonin function and melatonin-synthesis pathways and provide a new viewpoint for melatonin applications in the edible-medicinal fungus.

Keywords: melatonin, Tolypocladium guangdongense, quantification analysis, biosynthetic pathway, resistance to abiotic stressors, primordial formation

\section{INTRODUCTION}

Melatonin [N-acetyl-3-(2-aminoethyl)-5-methoxyindole] is an indoleamine that was first reported as a neurohormone in the bovine pineal gland (Lerner et al., 1958). In 1995, it was documented that melatonin can be synthesized in many edible plants, such as tomatoes, bananas, cucumbers, and potatoes (Dubbels et al., 1995; Hattori et al., 1995), which opened the avenues of investigation 
in melatonin research and attracted the attention of some researchers toward melatonin synthesis in extrapineal tissues. In the last two decades, accumulating evidences has shown that indoleamine is a ubiquitous in many organisms (Balzer and Hardeland, 1996; Hardeland and Poeggeler, 2003; RodriguezNaranjo et al., 2012). For fungi, intracellular melatonin was first observed in yeast cells arrested in minimal medium a study conducted by Sprenger et al. (1999). The existence of melatonin has been reported in some macro-fungi, such as Agaricus bisporus, Lentinula edodes, Pleurotus ostreatus, and Volvaria volvacea (Muszyńska et al., 2011; Muszyńska and Sułkowska-Ziaja, 2012; Gao et al., 2020).

In 1960, Axelord et al. first reported the classical pathway of melatonin biosynthesis in vertebrates. Using four enzymatic reactions, the precursor tryptophan was successively converted into 5-hydroxytryptophan (5HTryp), serotonin, and $\mathrm{N}$-acetylserotonin (NAS) as intermediates (Axelrod and Weissbach, 1960). The melatonin-synthesis pathway in plants differs from that in vertebrates (Arnao and Hernández-Ruiz, 2017). In plants, tryptophan is first converted into tryptamine by tryptophan decarboxylase (TDC) or into 5HTryp by tryptophan 5-hydroxylase (TPH). Second, tryptamine is catalyzed into $\mathrm{N}$-acetyltryptamine (NAT) and 5HT via serotonin $\mathrm{N}$-acetyltransferase (SNAT) and tryptamine 5-hydroxylase $(\mathrm{T} 5 \mathrm{H})$, respectively, whereas 5 HTryp is converted into serotonin by TDC. Third, T5H and SNAT catalyze the conversion of NAT and $5 \mathrm{HT}$, respectively, into $\mathrm{N}$-acetylserotonin (NAS), whereas serotonin is converted into 5-methoxytryptamine (5MT) by acetylserotonin O-methyltransferase (ASMT) or caffeic acid O-methyltransferase (COMT). Finally, NAS and 5MT are converted into melatonin by ASMT and SNAT, respectively. Muñiz-Calvo et al. (2019) analyzed the melatonin-synthesis pathway in Saccharomyces cerevisiae by quantifying changes in the levels of melatonin and its biosynthetic intermediates in yeast cells at different growth stages. The synthesis pathway in yeast was similar to that in plants, except that NAT were not detected. Nevertheless, the melatonin-synthesis pathway has not been elucidated in other fungi.

Melatonin plays an extremely important role as a multifunctional signaling molecule during responses to physiochemical processes. In animals and humans, melatonin has been shown to be mainly involved in photoperiodism and circadian rhythms and play various biological roles such as immunomodulatory, anticancer, anti-aging, and anti-obesity effects (Motilva et al., 2011; Salehi et al., 2019; Moradkhani et al., 2020). In plants, melatonin has shown many beneficial effects in term of seed germination, growth promotion, yield improvement, fruit preservation, and resistance against biotic and abiotic stresses (Posmyk et al., 2008; Wei et al., 2014; Aghdam et al., 2020; Nawaz et al., 2021). In Saccharomyces and non-conventional yeasts, melatonin can alleviate the damage induced by oxidative stress and ultraviolet stress (Vázquez et al., 2017, 2018; Bisquert et al., 2018). In addition, melatonin can regulate the fermentative capacity of yeasts by interacting with glycolytic proteins (Morcillo-Parra et al., 2020b). Several recent studies have reported that exogenous melatonin enhanced the cadmium tolerance in $V$. volvacea and retarded the senescence and the degree of cap browning in A. bisporus during the storage process (Gao et al., 2020; Li et al., 2021). In addition, limited information is available regarding the effects of melatonin on fungi.

Tolypocladium guangdongense, also named as Cordyceps guangdongensis, belongs to the same family as the traditional Chinese medicine Ophiocordyceps sinensis. The in vivo studies has shown that T. guangdongense markedly affected the growth of the influenza virus H9N2, reducing anti-inflammation in chronic bronchitis and alleviating chronic renal failure (Yan et al., 2010, 2012, 2014). These results indicate that $T$. guangdongense has the potential as an important economic fungus and can be applied in the functional food and healthcare industries. In addition, in our recent study, tryptophan and tryptamine levels peaked at the primordial $(\mathrm{P})$ stage (Wang et al., 2020a), and we detected melatonin and several intermediates when performing the metabolome analysis of T. guangdongense fruiting bodies (unpublished data), suggesting that the levels of melatonin and the associated intermediates notably differ during the growth and development process of T. guangdongense.

The aims of the current study were to quantify the levels of melatonin and its intermediates in T. guangdongense under abiotic stress [due to Congo red (CR), $\mathrm{H}_{2} \mathrm{O}_{2}$, heat, and cold stresses] and during different developmental stages. We also sought to identify the biosynthetic pathway of melatonin in T. guangdongense and analyzed the expression pattern of genes related to melatonin biosynthesis under different experimental conditions. Furthermore, we aimed to explore the potential role of melatonin in response to abiotic stress and in primordial formation. These results show the diversity of melatonin-synthesis pathways and its function and provide an important guiding significance of melatonin applications in the ediblemedicinal fungus.

\section{MATERIALS AND METHODS}

\section{Fungal Material and Treatments}

Tolypocladium guangdongense strain CCTCCM206051 was used in this study and deposited in the Agricultural Culture Collection of China. Several mycelial plugs were inoculated into $200 \mathrm{ml}$ improved CYM medium (20 g dextrose, $2 \mathrm{~g}$ yeast extract, $2 \mathrm{~g}$ peptone, $1 \mathrm{~g} \mathrm{KH}_{2} \mathrm{PO}_{4}, 1 \mathrm{~g} \mathrm{NaNO}, 0.5 \mathrm{~g} \mathrm{~K}_{2} \mathrm{HPO}_{4}$, $0.46 \mathrm{~g} \mathrm{MgSO}_{4} \cdot 7 \mathrm{H}_{2} \mathrm{O}$, and $0.1 \mathrm{~g}$ vitamin $\mathrm{B} 1$ in $11 \mathrm{ddH}_{2} \mathrm{O}$ ), and cultured for 10 days in an incubator shaker at 140 rotations/ min (rpm) and $23^{\circ} \mathrm{C}$. Next, mycelial pellets were transferred to PDA (potato dextrose agar) medium with or without $10 \mu \mathrm{M}$ $\mathrm{H}_{2} \mathrm{O}_{2}$ and $2 \mathrm{mg} / \mathrm{ml} \mathrm{CR}$ and incubated for 14 days at $23^{\circ} \mathrm{C}$. Mycelia cultured at $23^{\circ} \mathrm{C}$ for 4 days were subjected to 14 days $30^{\circ} \mathrm{C}$ or $14^{\circ} \mathrm{C}$ treatment as heat treatment (HT) and cold treatment (CT), respectively. In addition, the samples of mycelium (My), P, and young fruiting body (FB) were collected as described previously (Wang et al., 2020a). All the samples were immediately frozen in liquid nitrogen and then were preserved at $-80^{\circ} \mathrm{C}$ until metabolite, and RNA isolation were performed. 


\section{Quantification of Intracellular Melatonin Levels}

Samples $(0.1 \mathrm{~g})$ from different experimental conditions were homogenized in $1 \mathrm{ml}$ of a methanol/water $(50: 50, \mathrm{v} / \mathrm{v})$ mixture with $1.0 \mathrm{ng} / \mathrm{g}\left[{ }^{2} \mathrm{H}_{4}\right]$ MLT as an internal standard. After vortexinduced vibration for $15 \mathrm{~min}$, the mixture was centrifuged at $12,000 \mathrm{rpm}$ for $5 \mathrm{~min}$ at $4^{\circ} \mathrm{C}$, and the supernatant was collected. This procedure was repeated once. Both supernatants were merged and evaporated under a mild nitrogen stream. Then, the evaporated samples were re-dissolved in $100 \mu \mathrm{l}$ methanol/ water $(50: 50, \mathrm{v} / \mathrm{v})$ mixture. After $5 \mathrm{~min}$ vortex-induced vibration, the mixture was centrifuged at $12,000 \mathrm{rpm}$ for $5 \mathrm{~min}$ and filtered using a $0.22 \mu \mathrm{m}$ filter membrane. Intracellular melatonin levels were quantified by Wuhan Metware Biotechnology Co., Ltd. (Wuhan, China) using an liquid chromatography-tandem mass spectrometry (LC-MS/MS) platform (Waters Iclass-AB Sciex QTRAP 6500+) platform as described by Ye et al. (2017).

\section{Qualification of Levels of Indolic Compounds Related to Melatonin Biosynthesis}

The indolic compounds tryptophan, tryptamine, 5HTryp, NAT, serotonin, NAS, and 5MT were purchased from Sigma-Aldrich (Madrid, Spain) and Shanghai Yuanye Bio-Technology Co., Ltd (China). These indolic compounds were extracted using the method described in our previous study (Wang et al., 2020a). Briefly, sample tissues $(0.5 \mathrm{~g})$ from the $\mathrm{CR}$ and control (CK) groups was finely ground, followed by extraction in an $1 \mathrm{ml}$ buffer containing $50 \%$ methanol and $0.1 \%$ formic acid for $15 \mathrm{~min}$ in a shaker. After ultrasound treatment for $30 \mathrm{~min}$ in an ice bath, the mixtures were incubated at $4^{\circ} \mathrm{C}$ for $30 \mathrm{~min}$ and then centrifuged for $20 \mathrm{~min}$ at $12,000 \mathrm{rpm}$. The extracts were filtered through a $0.3 \mu \mathrm{m}$ filter membrane and were analyzed using LC-MS/MS platform (Waters Iclass-AB Sciex 6500) platform at Beijing Genomics Institute (BGI)-Shenzhen (Wuhan, China) as described by Sara et al. (2019).

\section{Expression Patterns of Genes Related to Melatonin Biosynthesis Under Different Conditions}

Genes related to melatonin biosynthesis in T. guangdongense were predicted by comparing the amino acid sequences with those of Oryza sativa and Mus musculus in T. guangdongense genome (BioProject: PRJNA399600). Tissue samples collected from $\mathrm{My}, \mathrm{P}$, and $\mathrm{FB}$ stages and the different periods of $\mathrm{CR}$ stress (6h, $12 \mathrm{~h}, 1$ days, 2 days, 4 days, 8 days, and 14 days) and were used for RNA isolation using the OminiPlant RNA Kit (DNase I; CWBIO, Beijing, China). The mRNA was reverse transcribed into cDNA and used for quantitative reverse transcriptase-polymerase chain reaction (qRT-PCR) analysis of genes related to melatonin biosynthesis. The gene-specific primers used for gene quantification were designed using Primer Primer5.0, and their sequences were shown in Table_S1. Histone H4 and the vacuolar protein sorting gene VPS were used as the internal control genes. The qRT-PCR reaction was performed using an Applied Biosystems ABI 7500 instrument (ABI, Foster
City, California, United States) and three biological and technical repeats. PCR reaction was performed with an initial denaturation for $3 \mathrm{~min}$ at $95^{\circ} \mathrm{C}$, followed by 40 cycles of $10 \mathrm{~s}$ at $95^{\circ} \mathrm{C}$ and annealing at $60^{\circ} \mathrm{C}$ for $20 \mathrm{~s}$. The relative expression levels of the target genes were calculated using the $2^{-\Delta \Delta \mathrm{Ct}}$ method (Wang et al., 2020b).

\section{Effects of Exogenous Melatonin on Tolypocladium guangdongense Under Different Conditions}

Ten micrometer $\mathrm{H}_{2} \mathrm{O}_{2}, 2 \mathrm{mg} / \mathrm{ml} \mathrm{CR}$, and $10 \mu \mathrm{M}$ melatonin were added to PDA medium at approximately $55^{\circ} \mathrm{C}$. Mycelial pellets activated for 10 days were inoculated into PDA medium and cultured at $23^{\circ} \mathrm{C}$. For the $\mathrm{HT}$ and CT groups, the PDA plates containing $10 \mu \mathrm{M}$ melatonin were placed in an incubator at $30^{\circ} \mathrm{C}$ and $14^{\circ} \mathrm{C}$. After 24 days of culture, the diameters of the fungal colonies were measured to assess the effects of melatonin on $T$. guangdongense mycelia under different experimental conditions. In addition, the groups containing $10 \mu \mathrm{M}$ melatonin were transferred into the cultivation room at a temperature of approximately $23^{\circ} \mathrm{C}$ and relative humidity of $60-70 \%$. Meanwhile, the mycelial pellets were subjected to treatment of $10 \mu \mathrm{M}$ melatonin, and then were inoculated in rice medium. After 15 days culture, they were transferred into the cultivation room at a temperature of approximately $23^{\circ} \mathrm{C}$ and relative humidity of $60-70 \%$. The mycelia were illuminated (500lx) for $10 \mathrm{~h}$ each day to explore the effect of melatonin on primordial formation.

\section{Statistical Analysis}

Significant differences were determined using the Statistical Package for the Social Sciences program (SPSS 22.0, SPSS Inc., Chicago, IL, United States). Error bars represent as the standard deviation from the mean of 10 independent replications in every group. Lowercase letters and asterisks were used to refer to significant differences between each group and the control group (CK).

\section{RESULTS AND DISCUSSION}

\section{Experimental Factors Affected Production of Intracellular Melatonin}

The levels of endogenous melatonin are regulated by multiple environmental factors. For example, endogenous melatonin synthesis was promoted by salt stress and a high temperature in sunflower and Arabidopsis seedlings (Mukherjee et al., 2014; Shi et al., 2015). In S. cerevisiae, a low temperature and low sugar level delayed the peaking of intracellular melatonin levels, whereas melatonin synthesis production was significantly promoted at $12^{\circ} \mathrm{C}$ (Wang et al., 2016; Morcillo-Parra et al., 2020a). To determine whether the intracellular melatonin levels of T. guangdongense markedly differ under different experimental conditions, T. guangdongense samples from three different developmental stages and four different abiotic stresses were collected, and melatonin contents were measured by LC-MS/MS. 
As shown in Figure 1A, three types of abiotic stresses significantly promoted intracellular melatonin production, whereas $\mathrm{H}_{2} \mathrm{O}_{2}$ stress tended to a decrease trend in intracellular melatonin synthesis. Compared to the intracellular melatonin content of the CK, CR exhibited the highest increase level (approximately 55.6-fold), flowed by CT (approximately 10.6-fold), and HT (approximately 5.13-fold), which was similar to the increase of melatonin levels in $V$. volvacea under Cd stress and A. bisporus during low temperature storage (Aghdam et al., 2019; Gao et al., 2020). Compared to My stage, intracellular melatonin levels had approximate 16 -fold and 28 -fold increase at the $\mathrm{P}$ and FB stages, respectively (Figure 1B). These results suggested that intracellular melatonin may be involved in the primordial formation, fruiting body growth and the response of T. guangdongense to abiotic stresses, which need to be confirmed by further study.

\section{Analysis of Intermediates Levels and Expression Pattern of Genes Related to Melatonin Synthesis Under Different Experimental Conditions}

In S. cerevisiae, Muñiz-Calvo et al. (2019) deciphered the melatonin biosynthesis and metabolism based on the bioconversion of related metabolites. According to their results and metabolome analysis conducted in our laboratory, the intermediates related to melatonin biosynthesis were detected in the mycelia and fruiting bodies of $T$. guangdongense (unpublished data). To more accurately quantify their dynamic changes and identify the melatonin-synthesis pathway, biosynthetic intermediate levels were quantified in fungal samples collected after 14days CR stress and during different developmental stages. The amino acid sequences of proteins related to melatonin biosynthesis in O. sativa and M. musculus were characterized by BLAST analysis against the genome of
T. guagndongense. Proteins with an E-value of $<0.001$ and $>35 \%$ amino acid similarity were treated as the homologous proteins. In the genome of $T$. guangdongense, nine genes were predicted to be the homologous genes involved in melatonin biosynthesis (Supplementary Table S1).

Our LC-MS/MS analysis revealed that the abundance of most intermediates markedly changed under CR condition compared to the corresponding levels found under normal culture condition (Figure 2A). The levels of tryptophan, the precursor of melatonin, were significantly increased in the CR medium. Tryptamine levels were not significantly different in the CR and CK media; however, the levels of four other intermediates exhibited a remarkable decease under CR condition. This phenomenon was inconsistent with the significant increase of melatonin content detected after being subjected to 14 days of CR stress. Therefore, the relative levels of these genes related to melatonin synthesis were analyzed during different periods of CR stress. As shown in Figure 2B, six genes were markedly upregulated in the early period but significantly downregulated in the late period, whereas the relative expression level of two genes showed an obvious down-regulation on days 8 and 14 . Specifically, five genes (TDC-1, TPH, T5H, SNAT-1, and ASMT2) were significantly upregulated during the first 8 days of $C R$ stress, but markedly downregulated on day 14 . The relative expression levels of $A A D C$ and ASMT-1 decreased significantly on days 8 and 14. TDC-2 was markedly upregulated on day 8 but downregulated after $12 \mathrm{~h}$ and on day 1. SNAT-2 was significantly downregulated on days 1,2 , and 8 , whereas was markedly upregulted after $6 \mathrm{~h}$ and on day 4 . From the perspective of gene expression pattern at different periods of CR stress, we speculated that the intermediates were synthesized and began to accumulate on day 8 or during an earlier period of CR stress, resulting in the accumulation and increase of melatonin at $14 \mathrm{~d}$ of $\mathrm{CR}$ stress.
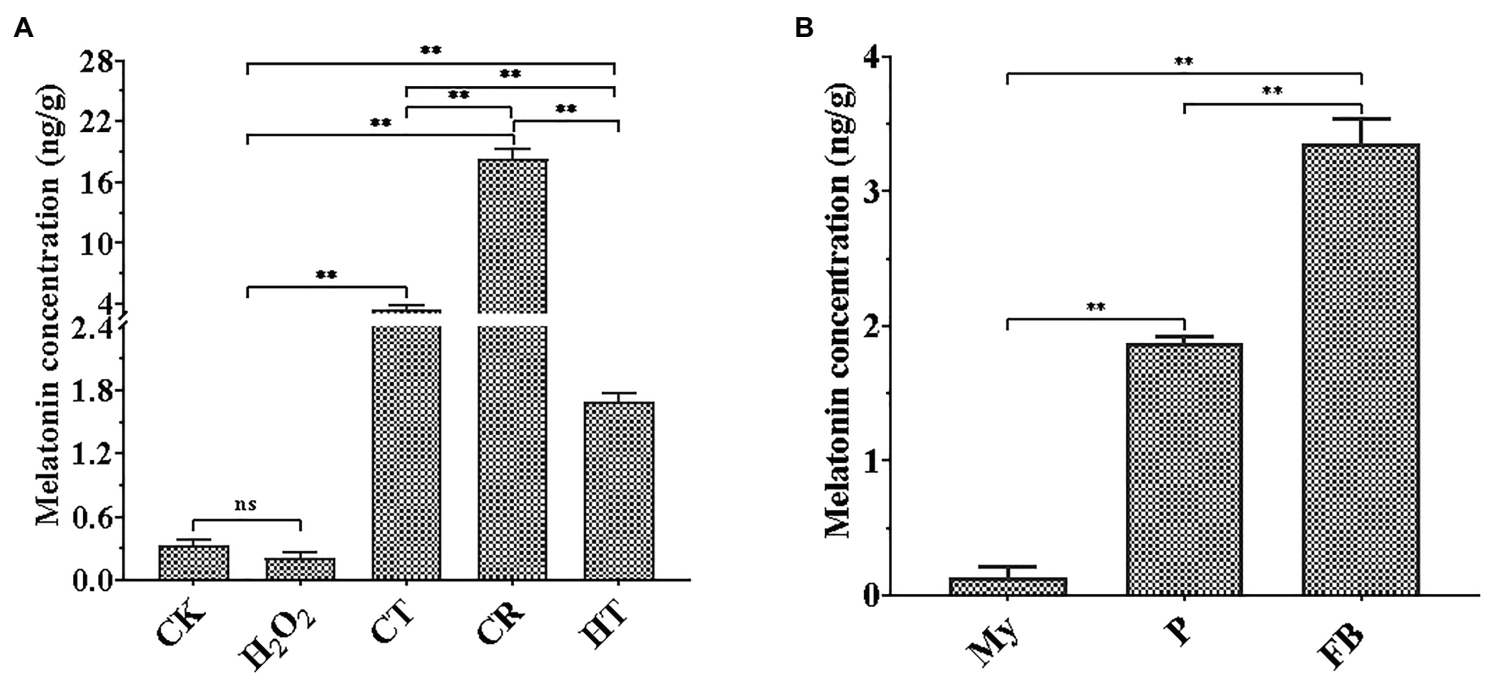

FIGURE 1 | Quantification of melatonin production in T. guangdongense at different abiotic stressors (A) and developmental stages (B). CK, mycelia untreated by several abiotic stresses; $\mathrm{H}_{2} \mathrm{O}_{2}, \mathrm{H}_{2} \mathrm{O}_{2}$ stress; $\mathrm{CT}$, cold stress; CR, congo red stress; My, mycelial stage; HT, heat stress; P, primordial stage; FB, fruiting body stage; *** highly significant differences $(p<0.01)$. 
A
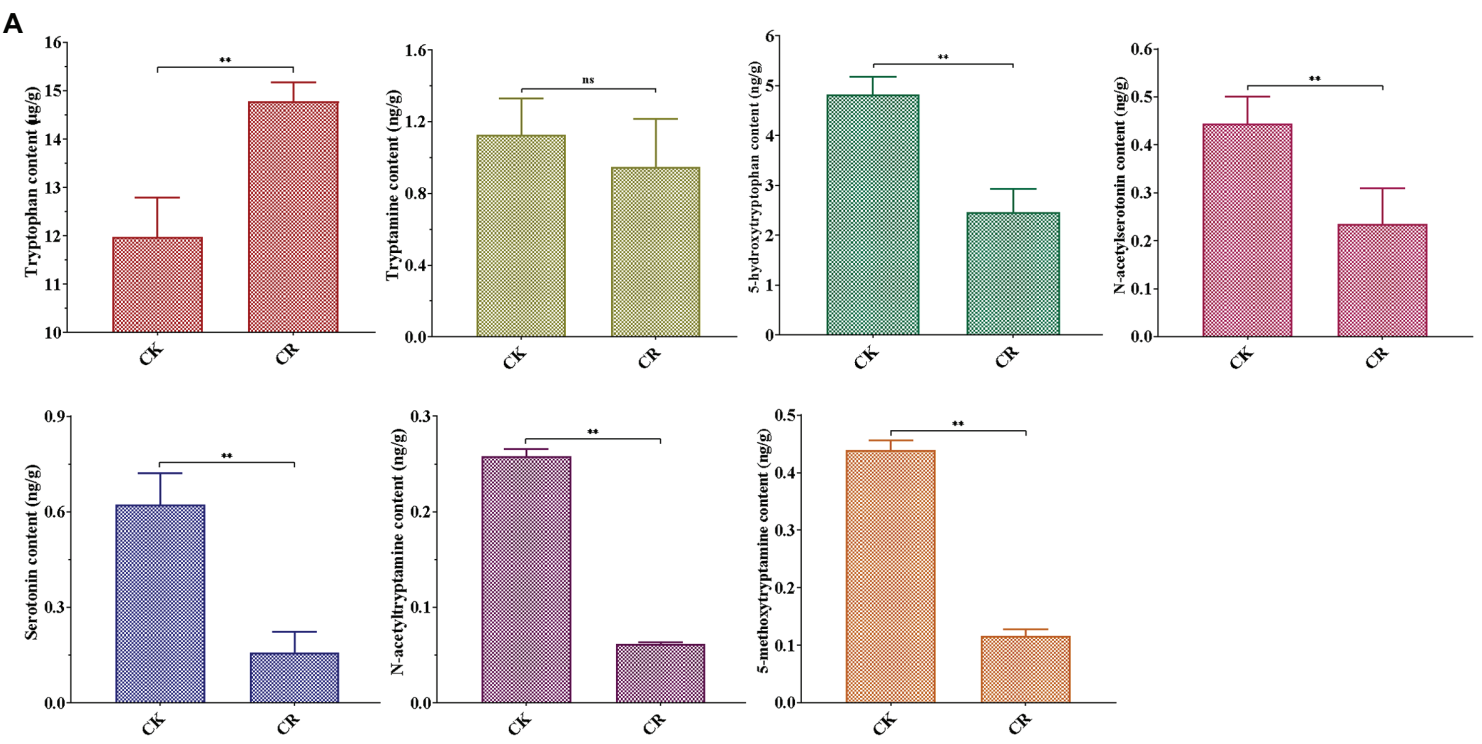

B
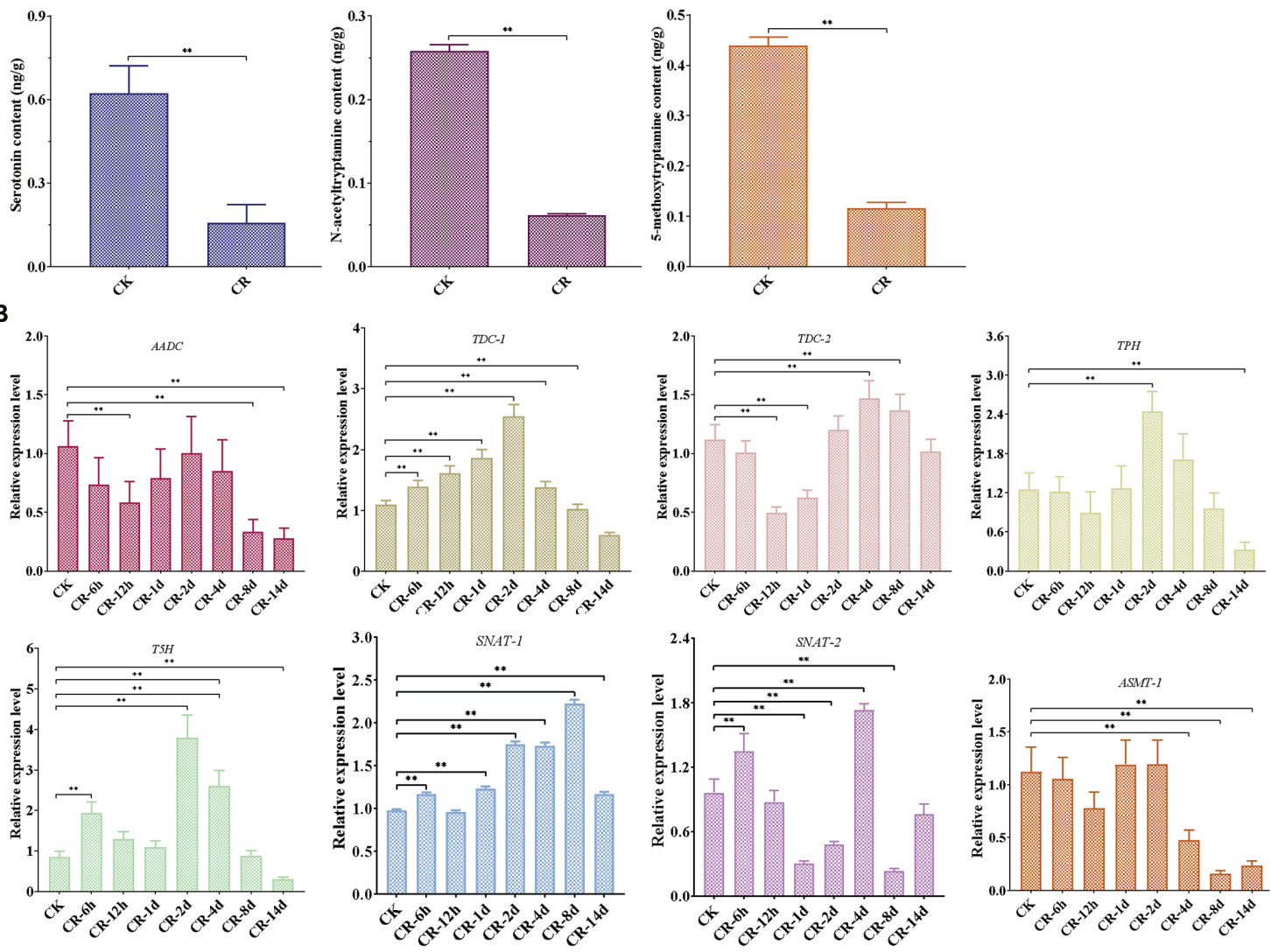

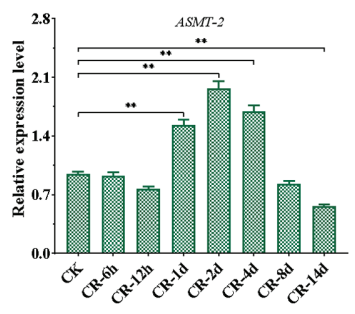

FIGURE 2 | Detection of melatonin-biosynthesis intermediates after $14 \mathrm{~d}$ CR treatment (A) and expression patterns of genes related to melatonin synthesis at different periods of $\mathrm{CR}$ stress (B) in T. guangdongense. CK, group at Oh.; CR, congo red; AADC, aromatic-L-amino-acid decarboxylase; TDC, tryptophan decarboxylase; TPH, tryptophan 5-hydroxylase; T5H, tryptamine 5-hydroxylase; SNAT, serotonin N-acetyltransferase; ASMT, acetylserotonin O-methyltransferase; ns, no significant difference. ${ }^{* *}$, highly significant differences $(p<0.01)$.

LC-MS/MS analysis showed that compared to My stage, the levels of five metabolites (tryptophan, tryptamine, 5HTryp, serotonin, and NAS) were significantly increased at the $\mathrm{P}$ and FB stages, whereas the levels of 5MT were markedly reduced (Figure 3A). In terms of the expression profile of genes related to melatonin synthesis, all the genes were significantly upregulated at $\mathrm{P}$ and $\mathrm{FB}$ stages compared to My stage (Figure 3B), being consistent to the higher levels of the intermediates at the $\mathrm{P}$ and FB stages. Comparing gene expression levels at the $\mathrm{P}$ and FB stages, the relative levels of six genes (AADC, TDC-2, TPH, 
T5H, SNAT-1, and ASMT-2) showed a significant upregulation at the FB stage, and two genes (TDC-1 and SNAT-2) were markedly upregulated at the $\mathrm{P}$ stage, in agreement with the quantitative analysis of the intermediate levels.
The synthesis pathway of melatonin was speculated in $T$. guangdongense, based on the above results and the related results from yeast and plants (Muñiz-Calvo et al., 2019; Nawaz et al., 2021). As shown in Figure 4, the putative
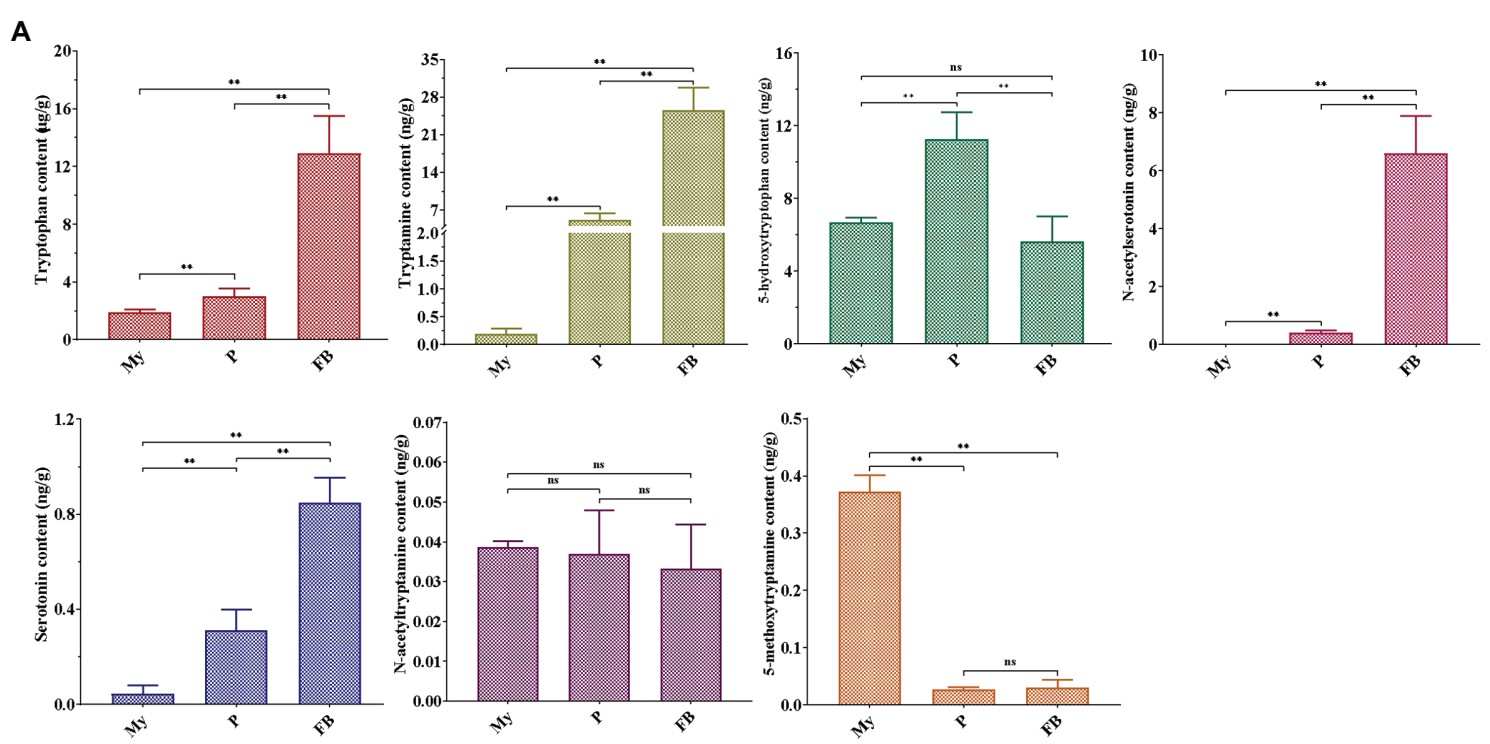

B
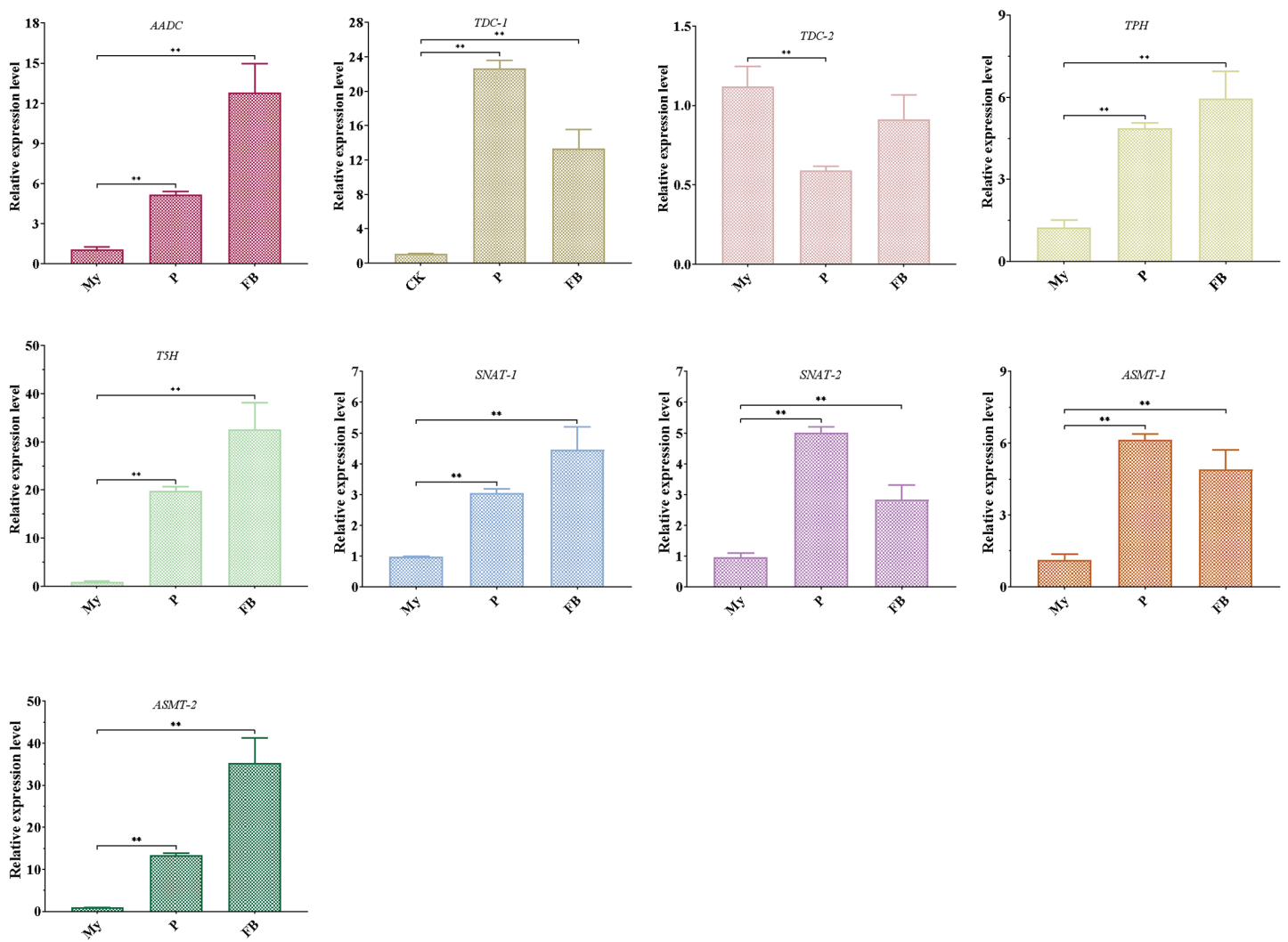

FIGURE 3 | Detection of melatonin-biosynthesis intermediates (A) and the expression pattern of genes related to melatonin synthesis (B) at different development stages of $T$. guangdongense. My, mycelial stage; P, primordial stage; FB, fruiting body stage; AADC, aromatic-L-amino-acid decarboxylase; TDC, tryptophan decarboxylase; TPH, tryptophan 5-hydroxylase; T5H, tryptamine 5-hydroxylase; SNAT, serotonin N-acetyltransferase; ASMT, acetylserotonin O-methyltransferase; ns, no significant difference. ${ }^{* *}$, highly significant differences $(p<0.01)$. 
melatonin-biosynthesis pathway in T. guangdongense was relatively complex compared to that in S. cerevisiae. In the first step of the proposed pathway, tryptophan was converted into tryptamine and 5HTryp by TDC/AADC and TPH, respectively. Later, this tryptamine was converted to NAT or serotonin by acetyltation (SNAT) and hydroxylation (T5H), respectively, whereas 5HTryp was converted to serotonin via 5-hydroxytryptophan carboxylation (TDC/AADC). Subsequently, serotonin was converted to NAS and 5MT by $\mathrm{N}$-acetylation (SNAT) and O-methylation (ASMT), respectively, whereas NAT was hydroxylated by $\mathrm{T} 5 \mathrm{H}$ to form NAS. Finally, T. guangdongense can covert NAS and five MT into melatonin by O-methylation (ASMT) and $\mathrm{N}$-acetylation (SNAT), respectively. According to the above results, it was suggested that serotonin and NAS were the main melatonin-synthesis intermediates, and the melatoninbiosynthesis pathway in T. guangdongense was markedly similar to that in plants (Nawaz et al., 2021).

\section{Exogenous Melatonin Promoted Mycelial Growth of Tolypocladium guangdongense Under Abiotic Stress Conditions}

By interacting with other signaling molecules, such as reactive oxygen species and nitric oxide, melatonin participates in various physiological reactions and enhances the resistance of plants to abiotic stress (Yu et al., 2018; Qiao et al., 2019). In fungi, exogenous melatonin enhanced the resistance of $S$. cerevisiae and $V$. volvacea against oxidative stress and cadmium stress, respectively (Vázquez et al., 2017; Gao et al., 2020). Therefore, colony diameters under different experimental

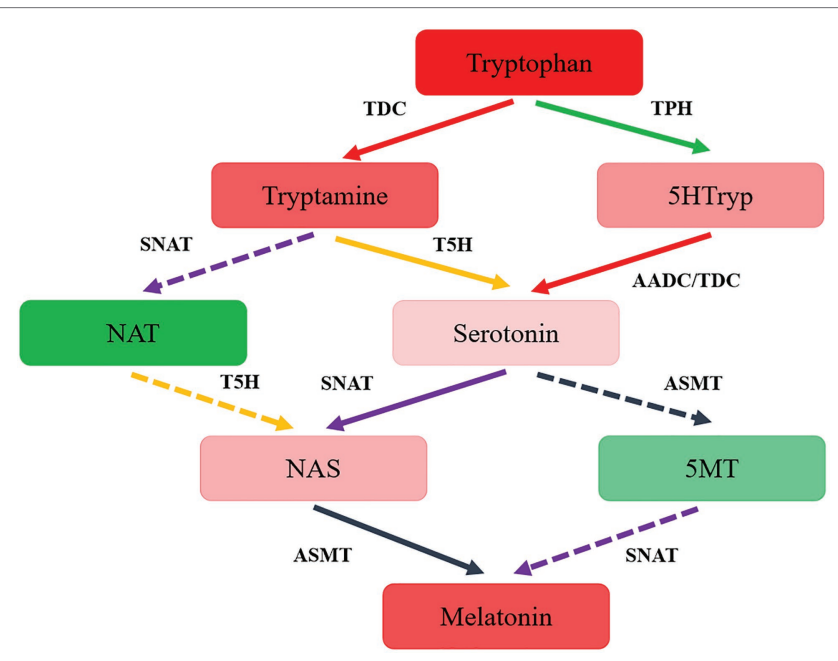

FIGURE 4 | The hypothesized pathway of melatonin biosynthesis in T. guangdongense. 5HTryp, 5-Hydroxytryptophan; NAT, N-acetyltryptamine; NAS, N-acetylserotonin; 5MT, 5-methoxytryptamine; AADC, aromatic-Lamino-acid decarboxylase; TDC, tryptophan decarboxylase; TPH, tryptophan 5-hydroxylase; $\mathrm{T} 5 \mathrm{H}$, tryptamine 5-hydroxylase; SNAT, serotonin

$\mathrm{N}$-acetyltransferase; ASMT, acetylserotonin O-methyltransferase; Full line represent the main procedures; colors, the higher degrees of red or green indicate the more or less levels of the intermediate contents, respectively. conditions were measured to evaluate the effect of exogenous melatonin on T. guangdongense mycelia.

Previously, it was found that treatment with $1 \mu \mathrm{M}$ and $1 \mathrm{M}$ melatonin obviously inhibited the mycelial growth of T. guangdongense, whereas no significant difference was found after treatment with $10 \mu \mathrm{M}$ or $100 \mu \mathrm{M}$ melatonin (Supplementary Figure S1). In addition, the mean of colony diameters in the $10 \mu \mathrm{M}$ melatonin group was the largest $(4.23 \mathrm{~cm})$. Therefore, $10 \mu \mathrm{M}$ melatonin was selected for this study. In terms of the four abiotic stresses applied, $2 \mathrm{mg} / \mathrm{ml} \mathrm{CR}$ showed an evident promotion of the mycelial growth, whereas the other three stresses had a significantly negative effect on the mycelial growth of $T$. guangdongense (Figure 5). The stimulatory effect of $2 \mathrm{mg} / \mathrm{ml} \mathrm{CR}$ was reduced in the presence of exogenous melatonin (Figure 5A). Nevertheless, exogenous melatonin promoted the mycelial growth of $T$. guangdongense and enhanced its resistance to heat, cold, and $\mathrm{H}_{2} \mathrm{O}_{2}$ stresses (Figures 5B-D), which was consistent with previous results (Wei et al., 2014; Yu et al., 2018; Nawaz et al., 2021). These results suggested that melatonin plays important roles in the response of T. guangdongense to abiotic stresses.

\section{Exogenous Melatonin Promoted Primordial Formation of Tolypocladium guangdongense}

As a growth-stimulating compound, melatonin is involved in several biological functions in plants such as the growth of roots and coleoptiles, seed germination, seedling growth, and senescence (Hernández-Ruiz et al., 2005; Wang et al., 2013; Arnao and Hernández-Ruiz, 2019). In this study, it was found that intracellular melatonin levels obviously increased at the $\mathrm{P}$ and FB stages (Figure 1B). Therefore, exogenous melatonin was added to PDA and rice medium to evaluate the effect of melatonin on primordial formation. Significantly more primordia were observed in the melatonin treatment group than those in the control group (Figure 6A). Additionally, the primordial density had an approximately one-fold increase in the melatonin treatment group (Figure 6B), indicating an important regulatory role in primordial formation in T. guangdongense. These results suggested that exogenous melatonin has the good potential for enhancing the cultivation of edible-medicinal mushroom.

\section{CONCLUSION}

In this study, we first quantified melatonin and the related biosynthetic intermediates in the macro-fungi. Several abiotic stressors promoted the synthesis of melatonin in $T$. guangdongense, and the melatonin and intermediate contents were relatively higher at the $\mathrm{P}$ and $\mathrm{FB}$ stages. However, the levels of most intermediates showed an obvious decrease after 14 days CR stress. After different periods of CR stress, most genes related to melatonin biosynthesis were markedly upregulated in the earlier period, but downregulated in the latter period, yet their relative expression levels showed a significant upregulation at the $\mathrm{P}$ and FB stages. On the basis of these results, a model of the melatonin-synthesis pathway was proposed in T. guangdongense. In addition, exogenous melatonin enhanced the resistance of $T$. guangdongense to 

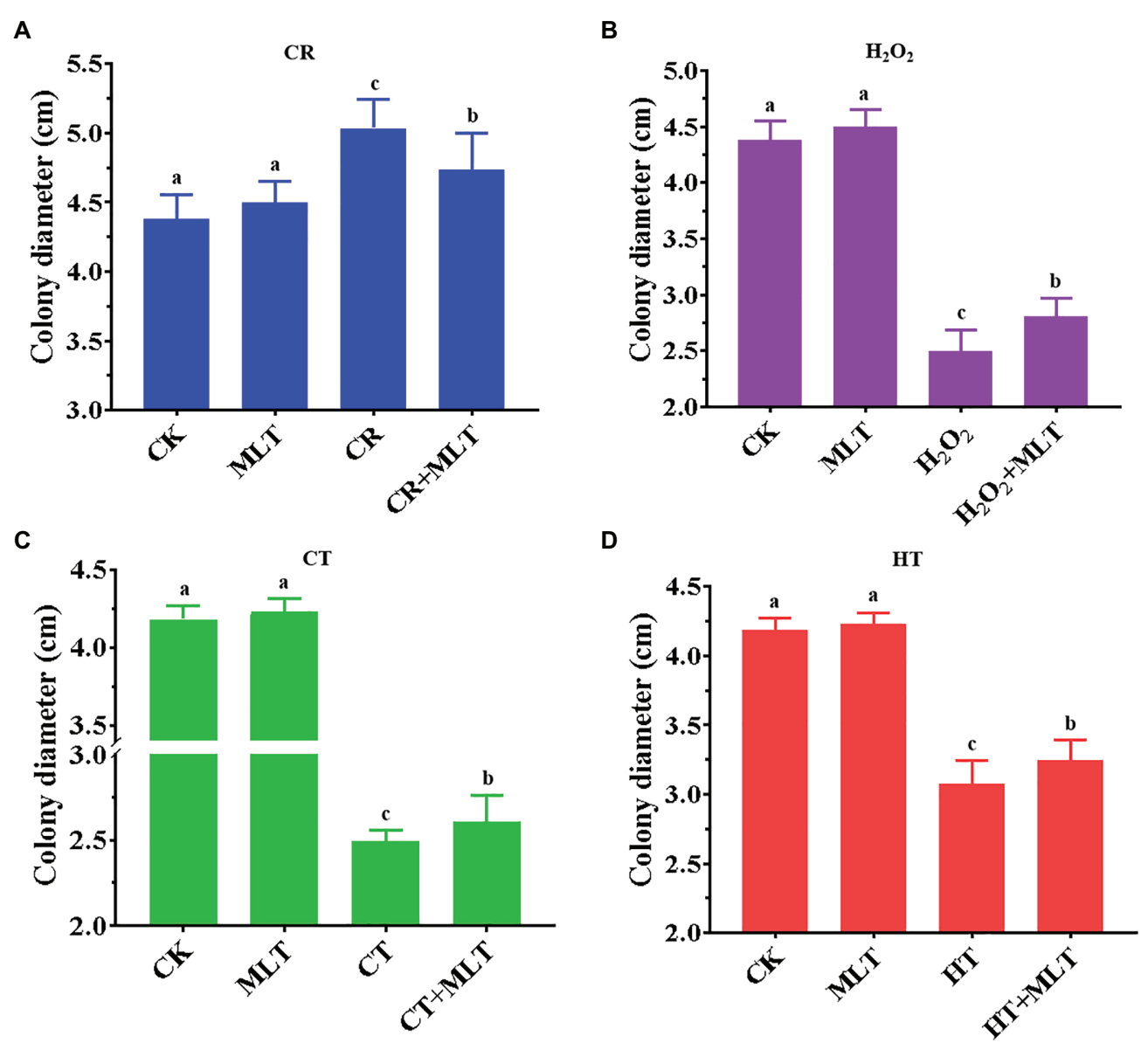

FIGURE 5 | Effect of exogenous melatonin on the mycelial growth of T. guangdongense under CR (A), H2O2 (B), CT (C) and HT stress (D), respectively. CT, cold stress; CR, cango red; HT, heat stress; MLT, melatonin; Lowercase letters represent significant difference $(\boldsymbol{D}<0.05)$.
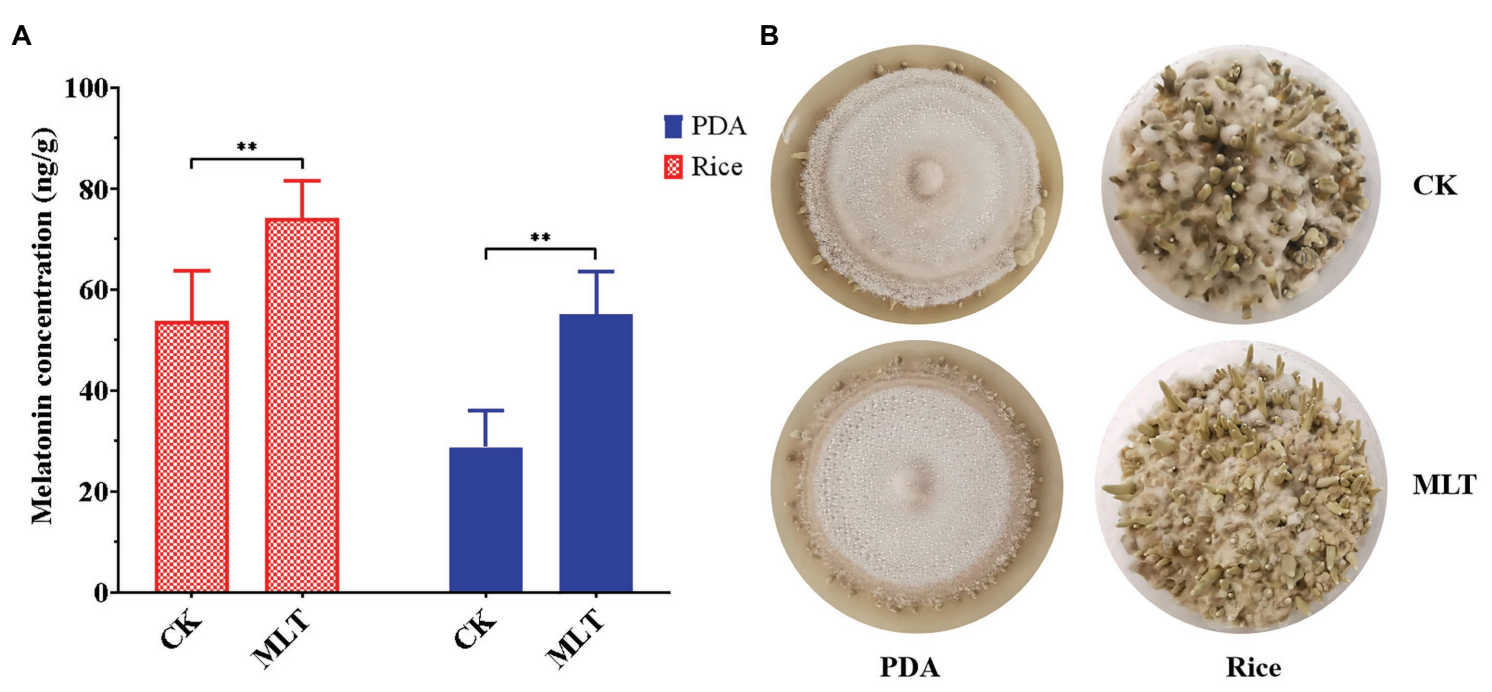

FIGURE 6 | Effect of exogenous melatonin on the primordial formation in T. guangdongense. (A) Primordial numbers of T. guangdongense on PDA and rice medium, respectively. (B) Primordial characteristics of $T$. guangdongense on PDA and rice medium. CK, group untreated by melatonin; MLT, group treated by $10 \mu \mathrm{M}$ melatonin. ${ }^{* *}$, highly significant differences $(p<0.01)$. 
the abiotic stress and promoted primordial formation. However, the underlying regulatory mechanism of melatonin remains unknown. In future study, it will be necessary to determine whether melatonin regulates the growth and development of fruiting bodies and the biosynthesis of active ingredients. In addition, molecular mechanisms regulating development, resistance to stress, and metabolite synthesis merit a comprehensive exploration.

\section{DATA AVAILABILITY STATEMENT}

The original contributions presented in the study are included in the article/Supplementary Material, further inquiries can be directed to the corresponding authors.

\section{AUTHOR CONTRIBUTIONS}

GW, CZ, TL, and WD conceptualized the study. GW and WD helped with the data curation. GW, XC, ML, and NZ contributed to the formal analysis. $\mathrm{XH}, \mathrm{TL}$, and WD were responsible for the funding acquisition. $\mathrm{ML}, \mathrm{CS}$, and $\mathrm{XC}$ carried out the investigation. GW, CZ, XC, ML, CS, and WD worked on the methodology. TL contributed to the resources. GW, CS, and $\mathrm{XC}$ were responsible for the validation. GW performed the visualization and wrote the original draft. $\mathrm{XH}, \mathrm{TL}, \mathrm{XC}$, and WD reviewed and edited the manuscript. All authors contributed to the article and approved the submitted version.

\section{REFERENCES}

Aghdam, M. S., Luo, Z., Jannatizadeh, A., and Farmani, B. (2019). Exogenous adenosine triphosphate application retards cap browning in Agaricus bisporus during low temperature storage. Food Chem. 293, 285-290. doi: 10.1016/j. foodchem.2019.05.002

Aghdam, M. S., Luo, Z., Li, L., Jannatizadeha, A., Fardc, J. R., and Pirzad, F. (2020). Melatonin treatment maintains nutraceutical properties of pomegranate fruits during cold storage. Food Chem. 303:125385. doi: 10.1016/j.foodchem.2019.125385

Arnao, M. B., and Hernández-Ruiz, J. (2017). Melatonin and its relationship to plant hormones. Ann. Bot. 121, 195-207. doi: 10.1093/aob/mcx114

Arnao, M. B., and Hernández-Ruiz, J. (2019). Role of melatonin to enhance phytoremediation capacity. Appl. Sci. 9:5293. doi: 10.3390/app9245293

Axelrod, J., and Weissbach, H. (1960). Enzymatic O-methylation of nacetylserotonin to melatonin. Science 131:1312. doi: 10.1126/science.131.3409.1312

Balzer, I., and Hardeland, R. (1996). Melatonin in algae and higher plants possible new roles as a phytohormone and antioxidant. Bot. Acta 109, 180-183. doi: 10.1111/j.1438-8677.1996.tb00560.x

Bisquert, R., Muñiz-Calvo, S., and Guillamón, J. M. (2018). Protective role of intracellular melatonin against oxidative stress and UV radiation in Saccharomyces cerevisiae. Front. Microbiol. 9:318. doi: 10.3389/fmicb.2018.00318

Dubbels, R., Reiter, R. J., Klenke, E., Goebel, A., Schnakenberg, E., Ehlers, C., et al. (1995). Melatonin in edible plants identified by radioimmunoassay and by high performance liquid chromatography mass spectrometry. J. Pineal Res. 18, 28-31. doi: 10.1111/j.1600-079X.1995.tb00136.x

Gao, Y., Wang, Y., Qian, J., Si, W., Tan, Q., Xu, J., et al. (2020). Melatonin enhances the cadmium tolerance of mushrooms through antioxidant-related metabolites and enzymes. Food Chem. 330:127263. doi: 10.1016/j. foodchem.2020.127263

Hardeland, R., and Poeggeler, B. (2003). Non-vertebrate melatonin. J. Pineal Res. 34, 233-241. doi: 10.1034/j.1600-079X.2003.00040.x

\section{FUNDING}

This research was funded by the National Natural Science Foundation of China (grant no. 31900015 and 31970024), Hunan Provincial Key Laboratory for Synthetic Biology of Traditional Chinese Medicine (no. HCSW2020-02), the Science and Technology Planning Project of Guangdong Province, China (nos. 2019B121202005), and the GDAS' Special Project of Science and Technology Development (no. 2020GDASYL20200103014).

\section{ACKNOWLEDGMENTS}

We sincerely thank Yuhua Gong in Huazhong Agricultural University for his suggestions and comments. We would also like to thank Editage (www.editage.cn) for English language editing.

\section{SUPPLEMENTARY MATERIAL}

The Supplementary Material for this article can be found online at: https://www.frontiersin.org/articles/10.3389/fmicb.2021.7461 41/full\#supplementary-material

Supplementary Figure S1 | Effect of different concentration melatonin on the mycelial growth of $T$. guangdongense.

Supplementary Table S1 | Information of genes related to melatonin biosynthesis.

Hattori, A., Migitaka, H., Iigo, M., Itoh, M., and Reiter, R. J. (1995). Identification of melatonin in plants and its effects on plasma melatonin levels and binding to melatonin receptors in vertebrates. Biochem. Mol. Biol. Int. 35, 627-634.

Hernández-Ruiz, J., Cano, A., and Arnao, M. B. (2005). Melatonin acts as a growth stimulating compound in some monocots pecies. J. Pineal Res. 39, 137-142. doi: 10.1111/j.1600-079X.2005.00226.x

Lerner, A. B., Case, J. D., Takahashi, Y., Lee, T. H., and Mori, W. (1958). Isolation of melatonin, the pineal gland factor that lightens melanocytes. $J$. Am. Chem. Soc. 80:2587. doi: 10.1021/ja01543a060

Li, L., Kitazawa, H., Zhang, X., Zhang, L., Sun, Y., Wang, X., et al. (2021). Melatonin retards senescence via regulation electron leakage of postharvest white mushroom (Agaricus bisporus). Food Chem. 340:127833. doi: 10.1016/j. foodchem.2020.127833

Moradkhani, F., Moloudizargari, M., Fallah, M., Asghari, N., Khoei, H. H., and Asghari, M. H. (2020). Immunoregulatory role of melatonin in cancer. J. Cell. Physiol. 235, 745-757. doi: 10.1002/jcp.29036

Morcillo-Parra, M. N., Beltran, G., Mas, A., and Torija, M. J. (2020a). Effect of several nutrients and environmental conditions on intracellular melatonin synthesis in Saccharomyces cerevisiae. Microorganisms 2020:853. doi: 10.3390/ microorganisms 8060853

Morcillo-Parra, M. A., Gonzalez, B., Beltran, G., Mas, A., and Torija, M. J. (2020b). Melatonin and glycolytic protein interactions are related to yeast fermentative capacity. Food Microbiol. 87:103398. doi: 10.1016/j.fm.2019.103398

Motilva, V., García-Mauriño, S., Talero, E., and Illanes, M. (2011). New paradigms in chronic intestinal inflammation and colon cancer: role of melatonin. J. Pineal Res. 51, 44-60. doi: 10.1111/j.1600-079X.2011.00915.x

Mukherjee, S., David, A., and Yadav, S. (2014). Salt stress-induced seedling growth inhibition coincides with differential distribution of serotonin and melatonin in sunflower seedling roots and cotyledons. Physiol. Plant. 152, 714-728. doi: 10.1111/ppl.12218 
Muñiz-Calvo, S., Bisquert, R., Fernández-Cruz, E., García-Parrilla, M. C., and Guillamón, J. M. (2019). Deciphering the melatonin metabolism in Saccharomyces cerevisiae by the bioconversion of related metabolites. J. Pineal Res. 66:e12554. doi: 10.1111/jpi.12554

Muszyńska, B., and Sułkowska-Ziaja, K. (2012). Analysis of indole compounds in edible Basidiomycota species after thermal processing. Food Chem. 132, 455-459. doi: 10.1016/j.foodchem.2011.11.021

Muszyńska, B., Sułkowska-Ziaja, K., and Ekiert, H. (2011). Indole compounds in some culinary-medicinal higher basidiomycetes from Poland. Int. J. Med. Mushrooms 13, 449-454. doi: 10.1615/IntJMedMushr.v13.i5.40

Nawaz, K., Chaudhary, R., Sarwar, A., Ahmad, B., Gul, A., Hano, C., et al. (2021). Melatonin as master regulator in plant growth, development and stress alleviator for sustainable agricultural production: current status and future perspectives. Sustainability 13:294. doi: 10.3390/su13010294

Posmyk, M. M., Kuran, H., Marciniak, K., and Janas, K. M. (2008). Presowing seed treatment with melatonin protects red cabbage seedlings against toxic copper ion concentrations. J. Pineal Res. 45, 24-31. doi: 10.1111/j.1600-079X.2007.00552.X

Qiao, Y., Yin, L., Wang, B., Ke, Q., Deng, X., and Wang, S. (2019). Melatonin promotes plant growth by increasing nitrogen uptake and assimilation under nitrogen deficient condition in winter wheat. Plant Physiol. Biochem. 139, 342-349. doi: 10.1016/j.plaphy.2019.03.037

Rodriguez-Naranjo, M. I., Torija, M. J., Mas, A., Cantos-Villar, E., and Garcia-Parrilla, M. C. (2012). Production of melatonin by Saccharomyces strains under growth and fermentation conditions. J. Pineal Res. 53, 219-224. doi: 10.1111/j.1600-079X.2012.00990.x

Salehi, B., Sharopov, F., Fokou, P. V. T., Kobylinska, A., and Iriti, M. (2019). Melatonin in medicinal and food plants: occurrence, bioavailability, and health potential for humans. Cells 8:681. doi: 10.3390/cells8070681

Sara, M., Bisquert, R., Edwin, F., María, C. G., José, M. G., et al. (2019). Deciphering the melatonin metabolism in Saccharomyces cerevisiae by the bioconversion of related metabolites. J. Pineal Res. 66:e12554. doi: 10.1111/ jpi.12554

Shi, H., Qian, Y., Tan, D. X., Reiter, R. J., and He, C. (2015). Melatonin induces the transcripts of CBF/DREB1s and their involvement in both abiotic and biotic stresses in Arabidopsis. J. Pineal Res. 59, 334-342. doi: 10.1111/ jpi.12262

Sprenger, J., Hardeland, R., Fuhrberg, B., and Han, S. Z. (1999). Melatonin and other 5-methoxylated indoles in yeast: presence in high concentrations and dependence on tryptophan availability. Cytologia 64, 209-213. doi: 10.1508/cytologia.64.209

Vázquez, J., González, B., Sempere, V., Mass, A., Torjia, M. J., and Beltran, G. (2017). Melatonin reduces oxidative stress damage induced by hydrogen peroxide in Saccharomyces cerevisiae. Front. Microbiol. 8:1066. doi: 10.3389/ fmicb.2017.01066

Vázquez, J., Grillitsch, K., Daum, G., Mass, A., Torjia, M. J., and Beltran, G. (2018). Melatonin minimizes the impact of oxidative stress induced by hydrogen peroxide in saccharomyces and non-conventional yeast. Front. Microbiol. 9:1933. doi: 10.3389/fmicb.2018.01933

Wang, G. Z., Cheng, H. J., Li, M., Zhang, C. H., Deng, W. Q., and Li, T. H. (2020b). Selection and validation of reliable reference genes for Tolypocladium guangdongense gene expression analysis under differentially developmental stages and temperature stresses. Gene 734:144380. doi: 10.1016/j.gene.2020
Wang, G. Z., Li, M., Zhang, C. H., Cheng, H. J., Gao, Y., Deng, W. Q., et al. (2020a). Transcriptome and proteome analyses reveal the regulatory networks and metabolite biosynthesis pathways during the development of Tolypocladium guangdongense. Comput. Struct. Biotechnol. J. 18, 2081-2094. doi: 10.1016/j. csbj.2020.07.014

Wang, P., Sun, X., Chang, C., Feng, F., Liang, D., and Cheng, L. (2013). Delay in leaf senescence of Malus hupehensis by long-term melatonin application is associated with its regulation of metabolic status and protein degradation. J. Pineal Res. 55, 424-434. doi: 10.1111/jpi.12091

Wang, C., Yin, L. Y., Shi, X. Y., Xiao, H., Kang, K., Liu, X. Y., et al. (2016). Effect of cultivar, temperature, and environmental conditions on the dynamic change of melatonin in mulberry fruit development and wine fermentation. J. Food Sci. 81, 958-967. doi: 10.1111/1750-3841.13263

Wei, W., Li, Q. T., Chu, Y. N., Reiter, R. J., Yu, X. M., Zhu, D. H., et al. (2014). Melatonin enhances plant growth and abiotic stress tolerance in soybean plants. J. Exp. Bot. 66, 695-707. doi: 10.1093/jxb/eru392

Yan, W. J., Li, T. H., and Jiang, Z. D. (2010). Antiviral activity of Cordyceps guangdongensis against influenza virus infections in mice. ACTA Edulis Fungi 17, 64-66.

Yan, W. J., Li, T. H., and Jiang, Z. D. (2012). Therapeutic effects of Cordyceps guangdongensis on chronic renal failure rats induced by adenine. Mycosystema 31, 432-442. doi: 10.13346/j.mycosystema.2012.03.017

Yan, W. J., Li, T. H., and Zhong, Z. (2014). Anti-inflammatory effect of a novel food Cordyceps guangdongensis on experimental rats with chronic bronchitis induced by tobacco smoking. Food Funct. 510, 2552-2557. doi: $10.1039 /$ c4fo00294f

Ye, T., Hao, Y. H., Yu, L., Shi, H., Reiter, R. J., and Feng, Y. Q. (2017). A simple, rapid method for determination of melatonin in plant tissues by UPLC coupled with high resolution Orbitrap mass spectrometry. Front. Plant Sci. 8:64. doi: 10.3389/fpls.2017.00064

Yu, Y., Wang, A., Li, X., Kou, M., Wang, W., Chen, X., et al. (2018). Melatoninstimulated triacylglycerol breakdown and energy turnover under salinity stress contributes to the maintenance of plasma membrane $\mathrm{H}^{+}$-ATPase activity and $\mathrm{K}^{+} / \mathrm{Na}^{+}$homeostasis in sweet potato. Front. Plant Sci. 9:256. doi: $10.3389 /$ fpls.2018.00256

Conflict of Interest: The authors declare that the research was conducted in the absence of any commercial or financial relationships that could be construed as a potential conflict of interest.

Publisher's Note: All claims expressed in this article are solely those of the authors and do not necessarily represent those of their affiliated organizations, or those of the publisher, the editors and the reviewers. Any product that may be evaluated in this article, or claim that may be made by its manufacturer, is not guaranteed or endorsed by the publisher.

Copyright (c) 2021 Wang, Chen, Zhang, Li, Sun, Zhan, Huang, Li and Deng. This is an open-access article distributed under the terms of the Creative Commons Attribution License (CC BY). The use, distribution or reproduction in other forums is permitted, provided the original author(s) and the copyright owner(s) are credited and that the original publication in this journal is cited, in accordance with accepted academic practice. No use, distribution or reproduction is permitted which does not comply with these terms. 